\title{
KSP inhibitor SB743921 induces death of multiple myeloma cells via inhibition of the NF- $\kappa B$ signaling pathway
}

\author{
In-Sung Song ${ }^{\#}$, Yu Jeong Jeong", Bayalagmaa Nyamaa, Seung Hun Jeong, Hyoung Kyu Kim, Nari Kim, \\ Kyung Soo Ko, Byoung Doo Rhee \& Jin Han * \\ National Research Laboratory for Mitochondrial Signaling, Department of Physiology, College of Medicine, Cardiovascular and Metabolic \\ Disease Center, Inje University, Busan 47392, Korea
}

\begin{abstract}
SB743921 is a potent inhibitor of the spindle protein kinesin and is being investigated in ongoing clinical trials for the treatment of myeloma. However, little is known about the molecular events underlying the induction of cell death in multiple myeloma (MM) by SB743921, alone or in combination treatment. Here, we report that SB743921 induces mitochondria-mediated cell death via inhibition of the NF-KB signaling pathway, but does not cause cell cycle arrest in KMS20 MM cells. SB743921-mediated inhibition of the NF-KB pathway results in reduced expression of SOD2 and Mcl-1, leading to mitochondrial dysfunction. We also found that combination treatment with SB743921 and bortezomib induces death in bortezomib-resistant KMS20 cells. Altogether, these data suggest that treatment with SB743921 alone or in combination with bortezomib offers excellent translational potential and promises to be a novel MM therapy. [BMB Reports 2015; 48(10): 571-576]
\end{abstract}

\section{INTRODUCTION}

Multiple myeloma (MM) is a type of hematological malignancy characterized by abnormal plasma cells in the bone marrow (1). In order to treat $\mathrm{MM}$, it is important to develop anticancer drugs targeting abnormal plasma cells. Since the introduction of combination therapy involving melphalan and prednisone, numerous multidrug chemotherapies employing agents such as dexamethasone, thalidomide, nitrosoureas, and bortezomib have been used to treat $\mathrm{MM}(2,3)$. However, many compounds have been reported to have resistance to anticancer activities when

*Corresponding author. Tel: +82-51-890-6727; Fax: +82-51-8945714; E-mail: phyhanj@inje.ac.kr

${ }^{\#}$ These authors contributed equally to this work.

http://dx.doi.org/10.5483/BMBRep.2015.48.10.015

Received 27 January 2015, Revised 26 February 2015, Accepted 11 March 2015

Keywords: SB743921, NF-кB, multiple myeloma, combination therapy, superoxide dismutase 2, Mcl-1 used as single treatment because of the multistep process of tumorigenesis $(4,5)$. Thus, it is required to develop novel compounds and strategies of optimized combinations of these drugs to improve the suvival of MM patients.

The spindle protein kinesin (KSP, human Eg5) is one of several microtubule-associated motor proteins required for proper spindle dynamics (6). KSP is essential for the formation of the bipolar spindle and proper segregation of sister chromatids during mitosis $(7,8)$. Furthermore, it is required for other critical physiological functions, such as intracellular transport and organelle positioning (9). Consequently, KSP-targeted novel antimitotic agents have been developed to cancer therapy, and several KSP inhibitors have entered clinical trials.

KSP inhibitors have been developed as a new generation of antimitotic agent with a novel mechanism of action for cancer therapy, and several KSP inhibitors have entered clinical trials. These KSP inhibitors, such as ispinesib (SB715992), ARRY-520, and MK0731, provide new opportunities for the development of hematological malignancy therapeutics. In particular, SB743921 is a selective KSP inhibitor that is being investigated in ongoing clinical trials for the treatment of myeloma, leukemia, and solid tumors $(10,11)$. However, little is known about the molecular events underlying the induction of cell death in multiple myeloma (MM) by SB743921, alone or in combination with other agents.

In this study, we first investigated the antimyeloma activity and mechanism of action for SB743921 in human MM cells. We found that this compound induces mitochondria-mediated cell death via inhibition of the NF-kB signaling pathway in these cells. To maximize the efficacy of SB743921 in treating MM, we investigated the antimyeloma activity by combined treatment with SB743921 and bortezomib in KMS20 bortezomib-resistant cells and found that the combination treatment induces cell death in KMS20 cells. Here, we present evidence that the mechanism of action of SB743921 is the inhibition of SOD2 and MCL-1 expression, which leads to bortezomib resistance.

\section{RESULTS}

SB743921 potently induces cell death in multiple myeloma cells

We first examined the capability of SB743921 to induce cell

ISSN: 1976-670X (electronic edition)

Copyright (C) 2015 by the The Korean Society for Biochemistry and Molecular Biology

(c) This is an open-access article distributed under the terms of the Creative Commons Attribution Non-Commercial License (http://creativecommons.org/licenses/by-nc/4.0) which permits unrestricted non-commercial use, distribution, and reproduction in any medium, provided the original work is properly cited. 
death using a CCK-8 assay. As shown in Fig. 1A, SB743921 induced cell death in a dose-dependent manner. SB743921-treated cells also displayed an abrupt time-dependent increase in cell death, visualized by annexin V/propidium iodide (PI) staining (Fig. 1B). Interestingly, SB743921 treatment induced cell death of more than $30 \%$ even at an early time point $(12 \mathrm{~h}$ ) and low concentration $(1 \mathrm{nM})$. Further, cell death was not significantly altered between $24 \mathrm{~h}$ and $48 \mathrm{~h}$. We next measured the change in cell death based on dose and duration of treatment using annexin V/PI staining (Fig. 1C). Cell death increased from $30 \%$ to $50 \%$ between $12 \mathrm{~h}$ and $24 \mathrm{~h}$. However, consistent with the data shown in Fig. 1A, cell death did not increase with an increase in dose from $1 \mathrm{nM}$ to $2 \mathrm{nM}$. Thus, the optimal dose and duration of treatment for SB743921-induced MM cell death were $1 \mathrm{nM}$ and $24 \mathrm{~h}$, respectively, and these conditions were used in subsequent experiments. We next used western blot analysis to determine whether SB743921 treatment modified caspase-3, caspase-8, and poly-(ADP-ribose) polymerase (PARP) levels in a time-dependent manner (Fig. 1D). Western bands for cleaved caspase-8, caspase-3, and PARP increased in density with SB743921 treatment in a time- dependent manner. Conversely, levels of procaspase-3, -8 , and -9 gradually decreased with SB743921 treatment. Next, we examined whether mitochondria were involved in SB743921-induced cell death. In the mitochondrial apoptotic pathway, the signal mediator between the cytosol and the mitochondrion is cytochrome $c$. Thus, cytochrome $c$ release was measured in SB743921-treated KMS20 cells (Fig. 1E). Cytochrome $c$ release into the cytosol was markedly increased in cells treated with SB743921. As a result of this release, caspase-3 cleavage was subsequently enhanced in KMS20 cells. Finally, we investigated whether SB743921 induces cell death in human primary bone marrow mononuclear cells. As shown in Fig. 1F, SB743921 did not induce cell death in these cells. Collectively, these results indicate that treatment with SB743921 alone specifically induces cell death in MM cells.

\section{SB743921 induces cell cycle arrest at early time points, but G2M arrest may not lead to cell death in MM cells}

To determine the effect of SB743921 on the cell cycle, we performed cell cycle analysis in KMS20 cells treated with $0.5 \mathrm{nM}$ and $1 \mathrm{nM} \mathrm{SB743921.} \mathrm{A} \mathrm{time-course} \mathrm{analysis} \mathrm{showed} \mathrm{that} \mathrm{cell} \mathrm{cycle} \mathrm{ar-}$ rest was detectable prior to cell death. In the case of cells treated with $0.5 \mathrm{nMSB} 743921, \mathrm{G} 2 \mathrm{M}$ arrest was detectable at $6 \mathrm{~h}$ and similarly decreased at $12 \mathrm{~h}$ and $24 \mathrm{~h}$, whereas cell death (subG1 phase) was detectable at $12 \mathrm{~h}$ (Fig. 2). However, G2M arrest in 1 nM-treated cells was detectable at $6 \mathrm{~h}$ and gradually decreased at $12 \mathrm{~h}-24 \mathrm{~h}$, whereas cell death (subG1 phase) was detectable at 12 $\mathrm{h}$ and more pronounced at $24 \mathrm{~h}$ (Fig. $2 \mathrm{~B}$ and C). This means that dead cells were primarily derived from the G2M-arrested population at a low concentration of SB743921, whereas cell death is independent of cell cycle status at a lethal dose. Next, we examined the level of p53 in SB743921-induced cell death. It has been suggested that an increase in $\mathrm{p} 53$ level is required for maximal cell sensitivity to microtubule-targeting agents $(12,13)$. However, p53 level was not increased in KMS20 cells treated with SB743921 (Fig. 2D). Collectively, our results suggest that SB743921 induces
A

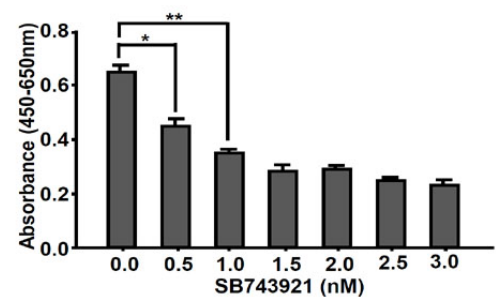

B
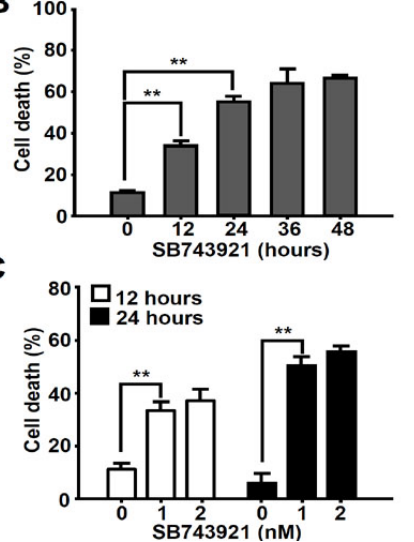

D

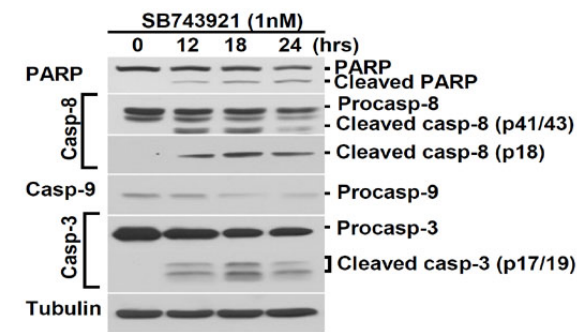

E

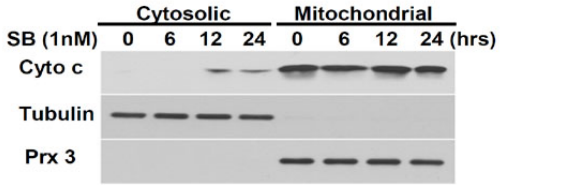

$\mathbf{F}$

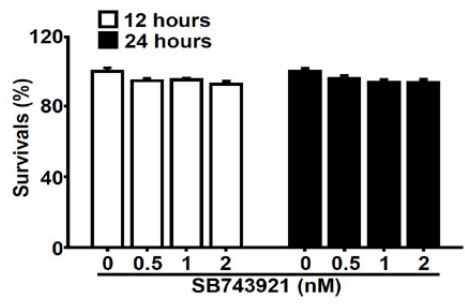

Fig. 1. SB743921 induces dose- and time-dependent cell death in MM cells. (A) Cells were treated with the indicated dose of SB743921, and cell death was determined at $24 \mathrm{~h}$ by CCK- 8 assay. (B) KMS20 cells were treated with $1 \mathrm{nM}$ SB743921 at the indicated time points, and cell death was determined by annexin V/PI staining. (C) KMS20 cells were treated with the indicated dose of SB743921, and cell death was determined at $12 \mathrm{~h}$ and $24 \mathrm{~h}$ by annexin $\mathrm{V} / \mathrm{PI}$ staining. (D) KMS20 cells were treated with $1 \mathrm{nMSB743921}$ for the indicated times and subjected to western blotting using the indicated antibodies. (E) KMS20 cells were treated with $1 \mathrm{nM}$ SB743921 for the indicated periods of times, and cell lysates were separated into cytosolic and mitochondrial fractions. Tubulin and peroxiredoxin 3 (Prx3) were used as cytosolic and mitochondrial markers, respectively. (F) To compare SB743921-induced cell death in a non-MM cells, human primary bone marrow mononuclear cells were treated with the indicated dose of SB743921 for the indicated times, and then subjected to CCK-8 assay to measurement of cell death. 
cell death independent of p53-dependent cell cycle arrest.

\section{SB743921 induces cell death via inhibition of the NF-кB signaling pathway}

$\mathrm{NF}-\kappa \mathrm{B}$ is aberrantly activated in a wide range of human cancers, including $\mathrm{MM}$, in which it promotes cell survival and malignancy by upregulating antiapoptotic genes $(14,15)$. Compelling evidence has established the paramount importance of aberrant NF- $\mathrm{kB}$ sig- naling in $\mathrm{MM}$ pathogenesis. To investigate the possible contribution of the NF- $\mathrm{KB}$ signaling pathway to this process, we investigated whether $\mathrm{I} \kappa \mathrm{B} \alpha$ phosphorylation is decreased by SB743921 treatment. In the case of KMS20 cells, NF-kB signaling was constitutively activated. As shown in Fig. $3 \mathrm{~A}$, I $\mathrm{I} B \alpha$ phosphorylation was clearly decreased in SB743921-treated KMS20 cells. Especially, the phosphorylation of $I \kappa B \alpha$ was dramatically decreased at $24 \mathrm{~h}$ after SB743921 treatment. Next, KMS20 cells were
A

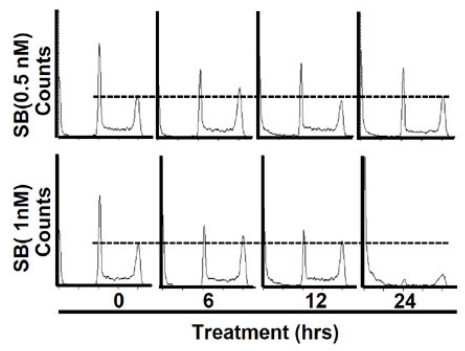

C

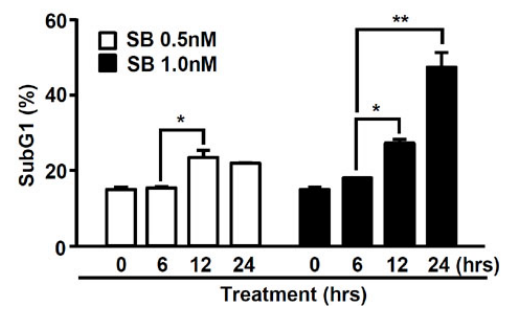

A
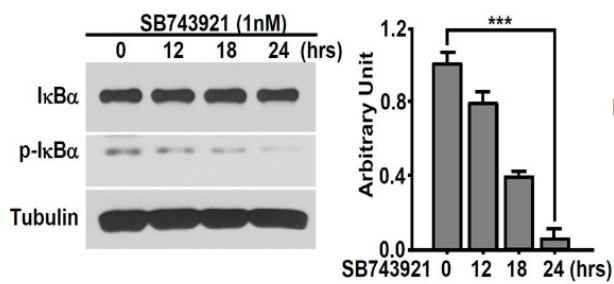

C

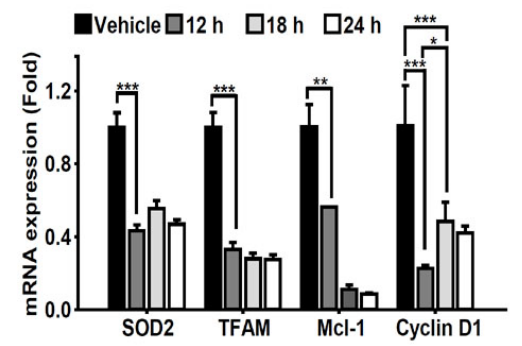

B

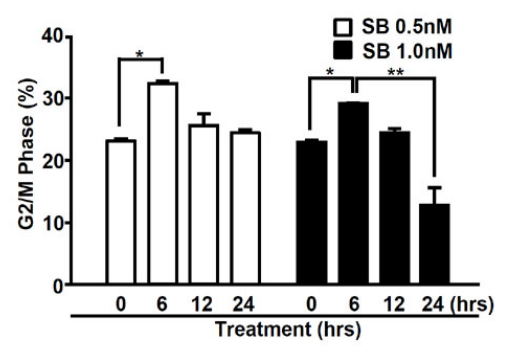

D

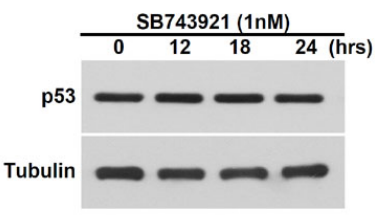

Fig. 2. SB743921 induces G2M arrest at an early time point prior to cell death. (A-C) KMS20 cells were incubated with the indicated doses of SB743921 for the indicated length of time, followed by FACS analysis of cell cycle distribution (A). Quantification of G2M arrest in (A) (B). Quantification of subG1 content in (A, C). (D) KMS20 cells were treated with $1 \mathrm{nM}$ SB743921 for the indicated length of time, and cells were analyzed by western blotting using anti-p53 and anti-tubulin antibodies.

B

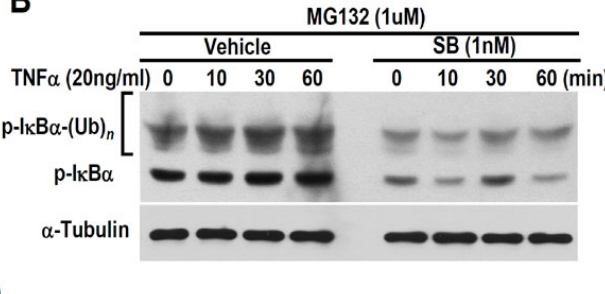

D

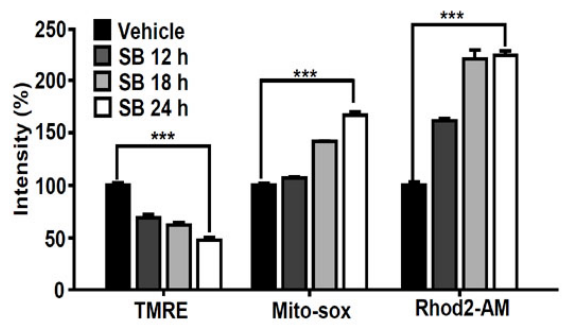

Fig. 3. SB743921 inhibits the NF-kB pathway, resulting in suppression of transcriptional activation of mitochondria-related genes, such as $\mathrm{MCl}-1$ and SOD2. (A) KMS20 cells were treated with SB743921 for the indicated times

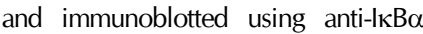
and anti-phospho-I $\mathrm{kB} \alpha$ antibodies. (B) SB743921-pretreated KMS20 cells for 12 hrs were stimulated with TNF- $\alpha$ (20 $\mathrm{ng} / \mathrm{ml}$ ) for the indicated times in the absence or presence of the proteasome inhibitor MG132 (1 hrs) and immunoblotted using an anti-phospho-IkB $\alpha$ antibodies. The polyubiquitinated species of phospho-lкB $\alpha$ are shown. (C) Relative expression levels of SOD2, TFAM, Mcl-1, and cyclin D1 in KMS20 cells pretreated with SB743921 for the indicated times were measured using qPCR. B2M served as the internal control. (D) Comparisons of mitochondrial calcium, ROS, and membrane potential in KMS20 cells treated with SB743921 for the indicated times generated using a FACSCanto II. 
A

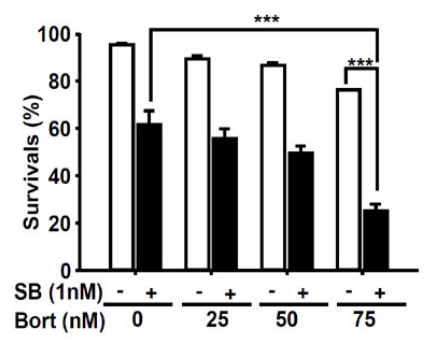

C

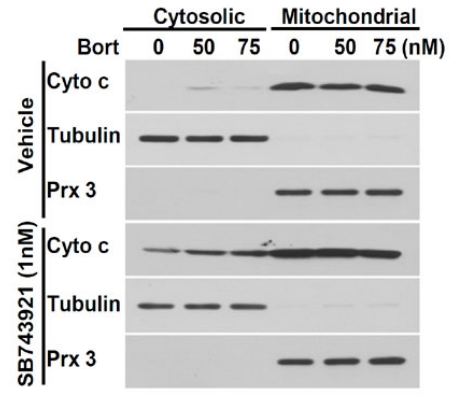

B

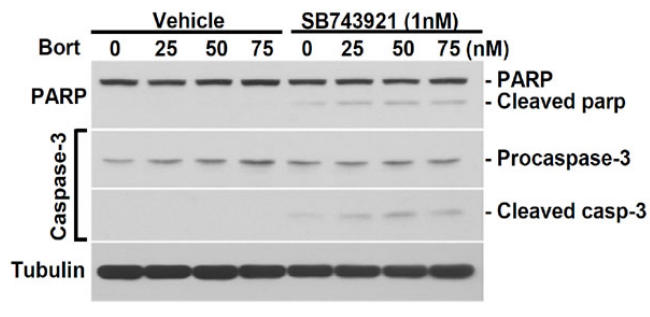

D

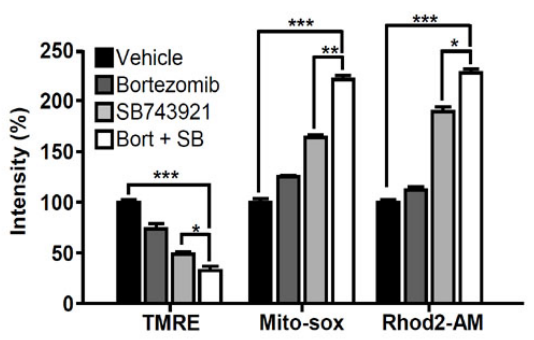

Fig. 4. Effects of combination treatment with SB743921 and bortezomib on apoptosis of KMS20 cells. (A) KMS20 cells were treated with SB743921 combined with bortezomib for $24 \mathrm{~h}$ at the indicated doses, and cell death was determined by annexin V/PI staining. (B) KMS20 cells were treated with SB743921 combined with bortezomib for $24 \mathrm{~h}$ at the indicated doses and analyzed by western blotting using the indicated antibodies. (C) KMS20 cells were treated with SB743921 combined with bortezomib for $24 \mathrm{~h}$, and the cells were separated into cytosolic and mitochondrial fractions. Tubulin and Prx3 served as cytosolic and mitochondrial markers, respectively. (D) Mitochondrial calcium, ROS, and membrane potential in KMS20 cells treated with SB743921 and bortezomib for the indicated times were measured using a FACSCanto II. co-treated with a proteasome inhibitor (MG-132) to determine whether the effect was due to a change in NF-kB activation (Fig. 3B). In the presence of MG132, SB743921-pretreated cells showed a dramatic reduction in $1 \kappa B \alpha$ phosphorylation. In contrast, the control cells showed a robust increase in the level of phosphorylated $I_{\kappa} \mathrm{B} \alpha$, including the polyubiquitinated species, consistent with the activation of endogenous NF- $\mathrm{BB}$ signaling. Additionally, induction of the endogenous NF- $\kappa \mathrm{B}$ target genes was examined in SB743921- treated cells. Levels of superoxide dismutase (SOD) 2; cyclin D1; TFAM; and an antiapoptotic Bcl-2 homologue, Mcl-1; were significantly reduced in SB743921-treated cells compared with control cells (Fig. 3C). Lastly, we examined whether SB743921-induced cell death was occurred through the mitochondrial dysfunction by the inhibition of the NF- $\kappa B$ signal pathway. As shown in Fig. 3D, mitochondrial ROS and calcium levels were increased by treatment with SB743921, and resulted in depolarization of the mitochondrial membrane potential. Collectively, these results show that SB743921 is an inhibitor of $\mathrm{NF}-\kappa \mathrm{B}$ signaling that mediates NF- $\mathrm{KB}$ transcriptional activation.

\section{Combined treatment with SB743921 and bortezomib overcomes bortezomib resistance}

In Fig. 1, we showed that cell death was induced by treament of SB743921. However, SB743921-induced cell death did not increase by more than $60 \%$, even at a concentration of $3 \mathrm{nM}$ or after $48 \mathrm{~h}$ of treatment in KMS20 cells. In a previous study, we reported that mitochondria play critical role in resisting cell death by bortezomib, and the potentiation of mitochondrial function resulted in the resistantance of KMS20 cells to bortezomib (16). Moreover, we have showed that alteration of mitochondria-related genes ex- pression led to changes in mitochondrial function and resistance or susceptibility to bortezomib; these alteration contributed to discriminative susceptibility or resistance to bortezomib in MM cells. Thus, the powerful activity of SB743921 in inducing cell death in MM (in even bortezomib-resistant cells) suggests the combination of SB743921 and bortezomib would increase therapeutic potency (Fig. 4). KMS20 cells were treated with 1 nM SB743921 and different concentrations of bortezomib for $24 \mathrm{~h}$ followed by labeling with annexin V-FITC/PI and flow cytometric analysis. As shown in Fig. 4, the combination of bortezomib with SB743921 showed strong additional cytotoxicity in KMS20 bortezomib-resistant cells, whereas the respective monotherapies had no or a moderately cytotoxic effect. To verify whether the cell death by combination treatment were induced by apoptosis, we performed immunoblot analysis with bortezomib or combination treatment in KMS20 cells. Immunoreative bands for cleaved caspase-3 and PARP were more intense in lysates of combination-treated cells than in those of cells treated with bortezomib alone (Fig. 4B). Finally, we examined whether mitochondria were involved in combination treatment-induced cell death. Cytochrome c release into the cytosol was markedly increased in cells treated with both compounds compared with those treated with bortezomib alone (Fig. 4C). Finally, we examined whether combination treatment with SB743921 and bortezomib induced mitochondrial dysfunction in KMS20 cells. As shown in Fig. 4D, mitochondrial ROS and calcium levels were significantly increased by combination treatment compared to single treatment, and resulted in the depolarization of mitochondrial membrane potential. Collectively, these results indicate that combination treatment with SB743921 and bortezomib induces a cell death mechanism in boretzomib-resistant KMS20 cells. 


\section{DISCUSSION}

SB743921 is being investigated in clinical trials for the treatment of myeloma, leukemia, and solid tumors, but its mechanism of action is unknown. Here, we show that SB743921 has significant cytotoxic activity in MM cells, supporting its potential use for therapeutic intervention in MM. Additionally, we demonstrated the induction of cell death specifically in MM cells, and not in human primary bone marrow cells (Fig. 1F). This study demonstrated that SB743921 inhibits the NF-KB signaling pathway without the impairing of cell cycle progression at an early time point, leading to MM cell death via activation of the mitochondria-mediated pathway. This effect is independent of both p53 level and cell cycle arrest. SB743921 strongly inhibited transcriptional activation of NF-kB target genes Mcl-1 and SOD2 $(15,17$, 18). Inhibition of the NF- $\kappa B$ survival pathway is possible and constitutes a promising candidate therapy that has no preclusive toxicity and could be of profound benefit for patients with MM (19).

Dipeptidyl-boronic acid derivative, bortezomib inhibits the proteasomes activity and is used in patients with relapsed/ refractory MM. Unfortunately, such MM patients have a low susceptibility to agents, and resistance have frequently occurred in early stage of treatment $(20,21)$. Here, we showed that the combination treatment of bortezomib and SB743921 induces cell death via inhibition of the NF-kB pathway in bortezomib-resistant MM cells. Human KMS20 MM cells were quite resistant at a high concentration bortezomib (22). In previous study, we reported that bortezomib resistance occurs because of changes in the expression of important mitochondrial genes, such as cyclophilin D, SOD2, and the mitochondrial calcium uniporter (16). Thus, SB743921-induced cell death may be the result of mitochondrial dysfunction via suppression of SOD2 and Mcl-1, induced by inhibition of the NF- $\mathrm{kB}$ pathway. However, the mechanism to NF-KB inhibition by SB743921 is not yet clear. Activation of the PI3K/AKT pathway, which is mostly related to signaling from major growth factors in $\mathrm{MM}$ (23), is also not yet verified in response to SB743921. Therefore, future research topics include examining potential candidates linking SB743921 to the IKK/NF$\kappa \mathrm{B}$ signaling cascades and PI3K/AKT activation.

In summary, our study reports that SB743921 alone and in combination with bortezomib induce cell death of myeloma cells as a novel therapeutic tool for relapsed/refractory MM. SB743921 alone and combined treatment with bortezomib facilitates reduction in protein levels of $\mathrm{MCl}-1$ and SOD2 due to the inhibition of the NF- $\mathrm{BB}$ pathway, and induces cell death in MM. Collectively, our results offer that treatment with SB743921, alone or in combination with bortezomib, offers excellent translational potential and novel strategies for MM therapy.

\section{MATERIALS AND METHODS}

Reagents, Cell culture, Measurement of cell death, Measurement of mitochondrial ROS, membrane potential $(\Delta \Psi \mathrm{m})$, and calcium concentration are described in the online data supplement, available at http://www.bmbreports.org/ .

\section{Western analysis and subcellular fractionation}

Cells were lysed in lysis buffer A [20 mM HEPES (pH 7.5), 1 mM EDTA, $2 \mathrm{mM}$ EGTA, $150 \mathrm{mM} \mathrm{NaCl}, 10 \%$ glycerol, $1 \%$ Triton $\mathrm{X}-100$, and protease cocktail I/II (Sigma-Aldrich)]. Proteins were separated by sodium dodecyl sulfate-polyacrylamide gel electrophoresis (SDS-PAGE) and transferred onto nitrocellulose membranes. Membranes were blocked in 5\% skim milk in 0.01 M Tris-buffered saline (pH 7.5) containing $0.5 \%$ Tween 20 and then incubated with the appropriate primary antibodies. For cytochrome c release after treatment with SB743921 alone or in combination with bortezomib, subcellular fractions were prepared using the ProteoExtract Subcellular Proteome Extraction Kit (Calbiochem, La Jolla, CA, USA). The purity of each fraction was verified using $\alpha$-tubulin and peroxiredoxin 3 (Prx3) as specific markers of cytosol and mitochondria, respectively (16).

\section{Measurement of cell cycle distribution}

Cell cycle distribution was determined by DNA staining with PI (Sigma-Aldrich). Cells $\left(1 \times 10^{6}\right)$ were collected and fixed in $70 \%$ ethanol. The cells were suspended in PI and simultaneously treated with RNase at $37^{\circ} \mathrm{C}$ for $30 \mathrm{~min}$ (24). The proportion of cells in each phase of the cell cycle was measured using a FACSCanto II flow cytometer (BD Biosciences).

\section{Quantitative PCR (qPCR)}

qPCR was performed using the first strand complementary DNA as template and specific primers for SOD2 (forward, 5'- ggaagccatcaaacgtgact-3'; reverse, 5'-acacatcaatccccagcagt-3'), Mcl-1 (forward, 5'-agaaagctgcatcgaaccat-3'; reverse, 5'-ccagctc ctactccagcaac-3'), TFAM (forward, 5'-ccgaggtggttttcatctgt-3'; reverse, 5'-tccgccctataagcatcttg-3'), B2M (forward, 5'-ctcgctccgtggccttag3'; reverse, 5'-caaatgcggcatcttcaa-3'), cyclin D1 (forward, 5'-caatgaccccgcacgatttc-3'; reverse, 5'-catggagggcggattggaa-3'). Amplification was carried out using the CFX96 ${ }^{\text {TM }}$ Real-Time PCR Detection System (Bio-Rad, Hercules, CA, USA) in 15-ul reaction mixtures containing 2 ul diluted DNA template, 2 pmol each primer, and 7.5 ul 2x SYBR ${ }^{\mathbb{R}}$ Premix Ex Taq ${ }^{\mathrm{TM}}$ (TaKaRa, Shiga, Japan). B2M was used as a control, and all reactions were performed in triplicate. Quantification of relative gene expression was analyzed using the $2^{-\Delta \Delta C T}$ method (25).

\section{Statistical analysis}

Data were analyzed using Student's t-test and SigmaPlot 8.0 software; $P$ values were derived to assess statistical significance, as follows: $* \mathrm{P}<0.05$; $* \mathrm{P}<0.01$; and ${ }^{* * * P}<0.001$. Data in all figures are expressed as the mean \pm standard deviation (SD) of three independent experiments.

\section{ACKNOWLEDGEMENTS}

This study was supported by a grant from the National R\&D Program for Cancer Control, Ministry for Health, Welfare and 
Family affairs, Republic of Korea (0920040), a Priority Research Centers Program through the National Research Foundation of Korea (NRF) funded by the Ministry of Education, Science, and Technology (2010-0020224), and a Basic Science Research Program through the National Research Foundation of Korea (NRF) funded by the Ministry of Education, Science, and Technology (2012R1A1A2041700).

\section{REFERENCES}

1. Kyle RA and Rajkumar SV (2004) Multiple myeloma. N Engl J Med 351, 1860-1873

2. San Miguel J, Blade J, Boccadoro M et al (2006) A practical update on the use of bortezomib in the management of multiple myeloma. Oncologist 11, 51-61

3. Chauhan D, Li G, Podar K et al (2004) Targeting mitochondria to overcome conventional and bortezomib/ proteasome inhibitor PS-341 resistance in multiple myeloma (MM) cells. Blood 104, 2458-2466

4. Ocio EM, Mateos MV, Maiso P, Pandiella A and San- Miguel JF (2008) New drugs in multiple myeloma: mechanisms of action and phase I/II clinical findings. Lancet Oncol 9, 1157-1165

5. Hanahan D and Weinberg RA (2000) The hallmarks of cancer. Cell 100, 57-70

6. Vale RD and Fletterick RJ (1997) The design plan of kinesin motors. Annu Rev Cell Dev Biol 13, 745-777

7. Enos AP and Morris NR (1990) Mutation of a gene that encodes a kinesin-like protein blocks nuclear division in A. nidulans. Cell 60, 1019-1027

8. Sawin KE and Mitchison TJ (1995) Mutations in the kinesin-like protein Eg5 disrupting localization to the mitotic spindle. Proc Natl Acad Sci U S A 92, 4289-4293

9. Rowinsky EK, Chaudhry V, Cornblath DR and Donehower RC (1993) Neurotoxicity of Taxol. J Natl Cancer Inst Monogr, 107-115

10. Talapatra SK, Anthony NG, Mackay SP and Kozielski F (2013) Mitotic kinesin Eg5 overcomes inhibition to the phase I/II clinical candidate SB743921 by an allosteric resistance mechanism. J Med Chem 56, 6317-6329

11. Yin $Y$, Sun $H, X u J$ et al (2014) Kinesin spindle protein inhibitor SB743921 induces mitotic arrest and apoptosis and overcomes imatinib resistance of chronic myeloid leukemia cells. Leuk Lymphoma, 1-8

12. Tan G, Heqing L, Jiangbo C et al (2002) Apoptosis induced by low-dose paclitaxel is associated with p53 upregulation in nasopharyngeal carcinoma cells. Int J Cancer 97, 168-172

13. Esteve MA, Carre M and Braguer D (2007) Microtubules in apoptosis induction: are they necessary? Curr Cancer Drug Targets 7, 713-729

14. Staudt LM (2010) Oncogenic activation of NF-kappaB. Cold Spring Harb Perspect Biol 2, a000109

15. DiDonato JA, Mercurio F and Karin M (2012) NF-kappaB and the link between inflammation and cancer. Immunol Rev 246, 379-400

16. Song IS, Kim HK, Lee SR et al (2013) Mitochondrial modulation decreases the bortezomib-resistance in multiple myeloma cells. Int J Cancer 133, 1357-1368

17. Park J, Bae EK, Lee C et al (2014) Establishment and characterization of bortezomib-resistant U266 cell line: constitutive activation of NF-kappaB-mediated cell signals and/or alterations of ubiquitylation-related genes reduce bortezomib-induced apoptosis. BMB Rep 47, 274-279

18. Yi MJ, Park SH, Cho HN et al (2002) Heat-shock protein 25 (Hspb1) regulates manganese superoxide dismutase through activation of Nfkb (NF-kappaB). Radiat Res 158, 641-649

19. Lam LT, Davis RE, Pierce J et al (2005) Small molecule inhibitors of IkappaB kinase are selectively toxic for subgroups of diffuse large B-cell lymphoma defined by gene expression profiling. Clin Cancer Res 11, 28-40

20. Richardson PG, Hideshima T, Mitsiades $C$ and Anderson KC (2007) The emerging role of novel therapies for the treatment of relapsed myeloma. J Natl Compr Canc Netw 5, 149-162

21. Lonial S, Waller EK, Richardson PG et al (2005) Risk factors and kinetics of thrombocytopenia associated with bortezomib for relapsed, refractory multiple myeloma. Blood 106, 3777-3784

22. Song IS, Jeong YJ, Jeong SH et al (2013) Combination treatment with 2-methoxyestradiol overcomes bortezomib resistance of multiple myeloma cells. Exp Mol Med 45, e50

23. Kong PJ, Byun JS, Lim SY et al (2008) Melatonin Induces Akt Phosphorylation through Melatonin Receptor- and PI3K-Dependent Pathways in Primary Astrocytes. Korean J Physiol Pharmacol 12, 37-41

24. Xie H, Hu J, Pan H, Lou Y, Lv P, Chen Y (2014) Adenovirus vector-mediated FAM176A overexpression induces cell death in human H1299 non-small cell lung cancer cells. BMB Rep 47, 104-109

25. Liu SJ, Yin CX, Ding MC, Xia SY, Shen QM, Wu JD (2014) Berberine suppresses in vitro migration of human aortic smooth muscle cells through the inhibitions of MMP-2/9, u-PA, AP-1, and NF- $\kappa$ B. BMB Rep 47, 388-392 